\title{
The angular distribution of mass by Bergman functions
}

Donald E. Marshall and Wayne Smith

Abstract. Let $\mathbb{D}=\{z:|z|<1\}$ be the unit disk in the complex plane and denote by $d \mathcal{A}$ two-dimensional Lebesgue measure on $\mathbb{D}$. For $\varepsilon>0$ we define $\Sigma_{\varepsilon}=\{z:|\arg z|<\varepsilon\}$. We prove that for every $\varepsilon>0$ there exists a $\delta>0$ such that if $f$ is analytic, univalent and area-integrable on $\mathbb{D}$, and $f(0)=0$, then

$$
\int_{f^{-1}\left(\Sigma_{\varepsilon}\right)}|f| d \mathcal{A}>\delta \int_{\mathbb{D}}|f| d \mathcal{A}
$$

This problem arose in connection with a characterization by Hamilton, Reich and Strebel of extremal dilatations for quasiconformal homeomorphisms of $\mathbb{D}$.

\section{Introduction.}

Let $\mathbb{D}=\{z:|z|<1\}$ be the unit disk in the complex plane and denote by $d \mathcal{A}$ two-dimensional Lebesgue measure on $\mathbb{D}$. The Bergman space $L_{a}^{1}$ consists of functions that are analytic on $\mathbb{D}$ and integrable with respect to $d \mathcal{A}$. It is a Banach space with norm

$$
\|f\|_{1}=\int_{\mathbb{D}}|f| d \mathcal{A} \text {. }
$$

Each $f \in L_{a}^{1}$ induces a Borel measure $\mu_{f}$ on the plane defined by

$$
\mu_{f}(E)=\int_{f^{-1} E}|f| d \mathcal{A}
$$


The problem considered in this paper concerns the angular distribution of mass by such a measure. For $\varepsilon>0$ we define

$$
\Sigma_{\varepsilon}=\{z:|\arg z|<\varepsilon\} .
$$

Theorem 1.1. For every $\varepsilon>0$ there exists a $\delta>0$ such that if $f \in L_{a}^{1}$ is univalent and $f(0)=0$, then

$$
\int_{f^{-1}\left(\Sigma_{\varepsilon}\right)}|f| d \mathcal{A}>\delta\|f\|_{1}
$$

Since (1.1) will then hold for all rotations of $\Sigma_{\varepsilon}$, Theorem 1.1 says that the measure $\mu_{f}$ cannot be too asymmetric. This theorem can not be extended to $L_{a}^{p}$ for any $p>1$; Example 4.3 at the end of the paper shows that (1.1) fails when $p>1,|f|$ is replaced by $|f|^{p}$, and $\|f\|_{1}$ is replaced by $\|f\|_{p}^{p}$.

As is explained below, it is known that there exist positive constants $C$ and $\eta$ such that

$$
C \int_{f^{-1}\left(\Sigma_{\pi / 2-\eta}\right)}|f| d \mathcal{A} \geq\|f\|_{1}, \quad \text { for all } f \in L_{a}^{1} \text { with } f(0)=0
$$

and it is an open problem to prove (1.1) without the restriction that $f$ be univalent. This is equivalent to a conjecture regarding quasiconformal mappings made by M. Ortel and the second author in [OS]. We now briefly review the relevant parts of this theory, and indicate the consequences that a solution to the open problem would have.

A bounded area-measurable function $\kappa$ on $\mathbb{D}$ with $\|\kappa\|_{\infty}<1$ is said to be a dilatation. It is a theorem in Ahlfors [A1] that to any dilatation $\kappa$ there is associated a unique quasiconformal homeomorphism $f^{\kappa}$ of $\mathbb{D}$ that fixes the points $1, i$, and -1 , and satisfies $\bar{\partial} f^{\kappa}=\kappa \partial f^{\kappa}$. We say that $\kappa$ is an extremal dilation if $\kappa$ is a dilatation and $\|\kappa\|_{\infty} \leq\left\|\kappa_{1}\right\|_{\infty}$ whenever $f^{\kappa}\left(e^{i \theta}\right)=f^{\kappa_{1}}\left(e^{i \theta}\right),-\pi<\theta \leq \pi$. The following characterization of extremal dilatations is due to R. Hamilton, S. L. Krushkal, E. Reich and K. Strebel.

Theorem $1.2([\mathrm{Ha}],[\mathrm{K}],[\mathrm{RS}])$. Suppose $\kappa$ is a dilatation. Then $\kappa$ is an extremal dilatation if and only if one of the following statements holds:

1) There exist $f \in L_{a}^{1}$ and $k \in[0,1)$ such that $\kappa(z)=k \overline{f(z)} /|f(z)|$ almost everywhere $d \mathcal{A}(z)$. 
2) There is a sequence $\left\{f_{n}\right\} \subset L_{a}^{1}$, converging to 0 uniformly on compact subsets of $\mathbb{D}$, such that $\left\|f_{n}\right\|_{1}=1$ and

$$
\lim _{n \rightarrow \infty} \int_{\mathbb{D}} f_{n} \kappa d \mathcal{A}=\|\kappa\|_{\infty} .
$$

Checking whether condition 2) holds for a particular dilatation can be difficult, and for this reason a more explicit characterization of extremal dilatations would be valuable. In this regard, we note it is not difficult to construct extremal dilatations that assume only countably many values and satisfy condition 2). Such a construction, based on an example in [OS], appears below. M. Ortel and the second author investigated the arguments of an extremal dilatation, and proved the following theorem.

Theorem 1.3 ([OS]). Suppose $\kappa$ is a bounded measurable function on $\mathbb{D}, \pi / 2<\theta<\pi / 2+\arctan \left(1 / 2 C_{0}\right)$, and $\kappa(z) \in \Sigma_{\theta} \cup\{0\}$ for almost all $z \in \mathbb{D}$. Then $\kappa$ is an extremal dilatation if and only if there exist $k \in[0,1)$ and $f \in L_{a}^{1}$ such that $\kappa(z)=k \overline{f(z)} /|f(z)|$ for almost all $z \in \mathbb{D}$.

Here $C_{0}$ is the infimum of those constants $C$ such that

$$
\int_{\mathbb{D}}|f| d \mathcal{A} \leq C \int_{\mathbb{D}}|\operatorname{Re} f| d \mathcal{A}
$$

for all $f \in L_{a}^{1}$ satisfying $\operatorname{Im} f(0)=0$. Subsequently, X. Huang $[\mathrm{Hu}]$ showed that this theorem remains valid when the number $\pi / 2+$ $\arctan \left(1 / 2 C_{0}\right)$ is replaced by the larger number $\pi / 2+\arcsin \left(1 /\left(2 C_{0}-\right.\right.$ $1))$. It was conjectured in [OS] that in the theorem, the number $\pi / 2+$ $\arctan \left(1 / 2 C_{0}\right)$ can in fact be replaced by $\pi$. In other words, if $\kappa$ is an extremal dilatation not of the form $k \bar{f} /|f|$, with $f \in L_{a}^{1}$, then the arguments of $\kappa$ were conjectured to be dense in the unit circle.

This conjecture is equivalent to the extension of Theorem 1.1 from univalent functions to all functions in $L_{a}^{1}$. To see this, first suppose that (1.1) holds for all $f \in L_{a}^{1}$ and that $\kappa$ is a dilatation satisfying

$$
\kappa(z) \in \mathbb{C} \backslash \Sigma_{2 \varepsilon},
$$

for almost all $z \in \mathbb{D}$. Let $f$ be such that $\|f\|_{1}=1$ and $f(0)=0$. If $f(z) \in \Sigma_{\varepsilon}$, then $f(z) \kappa(z) \in \mathbb{C} \backslash \Sigma_{\varepsilon}$. Thus

$$
\operatorname{Re} \int_{f^{-1}\left(\Sigma_{\varepsilon}\right)} f \kappa d \mathcal{A} \leq \cos (\varepsilon)\|\kappa\|_{\infty} \int_{f^{-1}\left(\Sigma_{\varepsilon}\right)}|f| d \mathcal{A},
$$


and so

$$
\begin{aligned}
\operatorname{Re} \int_{\mathbb{D}} f \kappa d \mathcal{A} & \leq\|\kappa\|_{\infty}+(\cos (\varepsilon)-1)\|\kappa\|_{\infty} \int_{f^{-1}\left(\Sigma_{\varepsilon}\right)}|f| d \mathcal{A} \\
& <\|\kappa\|_{\infty}+(\cos (\varepsilon)-1)\|\kappa\|_{\infty} \delta \\
& <\|\kappa\|_{\infty},
\end{aligned}
$$

where $\delta>0$ comes from (1.1). Thus, condition (2) of Theorem 1.2 can not hold and $\kappa$ is not extremal unless it is of the form $k \bar{f} /|f|$, where $f \in L_{a}^{1}$. Hence if the arguments of $\kappa$ are not dense in the circle, then condition 2) of Theorem 1.2 fails. For the converse, suppose there is a sequence $\left\{f_{n}\right\} \subset L_{a}^{1}$ with $\left\|f_{n}\right\|_{1}=1, f_{n}(0)=0$, and such that

$$
\lim _{n \rightarrow \infty} \int_{f_{n}^{-1}\left(\Sigma_{\varepsilon}\right)}\left|f_{n}\right| d \mathcal{A}=0
$$

It is easy to check that if $f$ is a normal limit of $\left\{f_{n}\right\}$, then

$$
\int_{f^{-1}\left(\Sigma_{\varepsilon}\right)}|f| d \mathcal{A}=0 .
$$

Since also $f(0)=0$, it follows that $f$ is identically 0 , and so $\left\{f_{n}\right\}$ converges to zero uniformly on compact subsets of $\mathbb{D}$. Then, by approximating $\overline{f_{n}} /\left|f_{n}\right|$ on an appropriate sequence of annuli in $\mathbb{D}$ while omitting values in $\Sigma_{\varepsilon}$, it is possible to construct a dilatation $\kappa$ that satisfies condition 2) of Theorem 1.2 and which assumes no values in $\Sigma_{\varepsilon}$.

Thus we have shown that the conjecture from [OS] of the density in the unit circle of the arguments of an extremal dilatation $\kappa$ not of the form $k \bar{f} /|f|$, with $f \in L_{a}^{1}$, is equivalent to the conjecture that the conclusion of Theorem 1.1 is valid for all $f \in L_{a}^{1}$ with $f(0)=0$. We also note that the argument sketched above, together with the theorem quoted from [OS], shows that there exist positive constants $C$ and $\eta$ such that (1.2) holds.

In the next section we collect facts and background material on the hyperbolic metric and harmonic measure that will be used to prove Theorem 1.1 in Section 3. Finally, some examples have been included in the last section. These examples illustrate how some of the difficulties encountered were addressed in the proof, and that Theorem 1.1 can not be extended to $L_{a}^{p}$ for any $p>1$. 


\section{Background.}

The main tools we will use in the proof of Theorem 1.1 are the hyperbolic distance and harmonic measure. The hyperbolic distance on $\mathbb{D}$ is defined by (see $[$ A2, p. 2])

$$
\rho_{\mathbb{D}}\left(z_{1}, z_{2}\right)=\inf \left\{\int_{\gamma} \frac{2|d z|}{1-|z|^{2}}: \gamma \text { is an arc in } \mathbb{D} \text { from } z_{1} \text { to } z_{2}\right\} .
$$

For example, the shortest distance from 0 to any other point is along a radius, and

$$
\rho_{\mathbb{D}}(0,|z|)=\log \left(\frac{1+|z|}{1-|z|}\right) .
$$

This distance is invariant under conformal self-maps of $\mathbb{D}$ and thus the hyperbolic geodesics are diameters of the disk together with circles orthogonal to the unit circle. This distance also transfers to a natural conformally invariant distance on any simply connected proper subset $G \subset \mathbb{C}$. If $\varphi: \mathbb{D} \longrightarrow G$ is any conformal map, the hyperbolic distance on $G$ is given by $\rho_{G}\left(w_{1}, w_{2}\right)=\rho_{\mathbb{D}}\left(z_{1}, z_{2}\right)$, where $w_{i}=\varphi\left(z_{i}\right)$ for $i=1,2$. The shortest arc in $\mathbb{D}$ from $z_{1}$ to $z_{2}$ is the arc of the unique circle orthogonal to $\partial \mathbb{D}$ passing through $z_{1}$ and $z_{2}$. The shortest arc in $G$ from $w_{1}$ to $w_{2}$ is the image of this arc in $\mathbb{D}$ by the map $\varphi$. If $E \subset G$, then the hyperbolic distance from $z_{1}$ to $E$ will be denoted by $\rho_{G}\left(z_{1}, E\right)$.

The harmonic measure of a set $E$ contained in the closure of a region $\Omega$ evaluated at $z \in \Omega$ is denoted by $\omega(z, E, \Omega)$. It is (roughly) the function which is harmonic on $\Omega \backslash E$, equal to 1 on $E$ and equal 0 on $\partial \Omega \backslash E$. See $[\mathrm{GM}]$ for a precise definition.

\subsection{Area Estimates.}

We can use both the hyperbolic distance and harmonic measure to estimate the Euclidean area $\mathcal{A}(E)$ of a measurable set $E \subset \mathbb{D}$ :

$$
\mathcal{A}(E) \leq C e^{-\rho_{\mathbb{D}}(0, E)} \omega(0, E, \mathbb{D}),
$$

for some universal constant $C<\infty$. To see this, let $E^{*}=\{z /|z|: z \in$ $E\}$ denote the radial projection of $E$ onto $\partial \mathbb{D}$. Then $E$ is contained in the set

$$
\left\{z \in \mathbb{D}: \rho_{\mathbb{D}}(0, z) \geq \rho_{\mathbb{D}}(0, E) \text { and } \frac{z}{|z|} \in E^{*}\right\}
$$


which has area at most $2 e^{-\rho_{\mathbb{D}}(0, E)}\left|E^{*}\right|$, where $\left|E^{*}\right|$ denotes the length of the projection $E^{*}$. Equation (2.1) now follows from Hall's Lemma [GM].

If $E$ is a hyperbolic ball, then a similar lower estimate is available for the area: If $E$ is a hyperbolic ball with hyperbolic radius at least $\rho_{0}$, then

$$
\mathcal{A}(E) \geq C\left(1-e^{-\rho_{0}}\right)^{2} e^{-\rho_{\mathbb{D}}(0, E)} \omega(0, E, \mathbb{D}),
$$

for some universal constant $C>0$. Each quantity in the right-hand side of (2.2) can be computed explicitly using conformal invariance. Another way to make the lower estimate in (2.2) is to set $d=\inf \{|z|: z \in E\}$, so that

$$
e^{-\rho_{\mathbb{D}}(0, E)}=\frac{1-d}{1+d} .
$$

Inequality (2.2) is easy to prove if $0 \in E$, so assume that $0 \notin E$, and let $\Gamma$ denote the circle orthogonal to the unit circle separating $E$ from 0 with $\rho_{\mathbb{D}}(0, \Gamma)=\rho_{\mathbb{D}}(0, E)$, and let $I$ denote the subarc of $\partial \mathbb{D}$ subtended by $\Gamma$ and separated from 0 by $\Gamma$. Then

$$
\omega(z, I, \mathbb{D}) \equiv \frac{1}{2}
$$

for all $z \in \Gamma$, and hence

$$
\omega(0, E, \mathbb{D}) \leq \omega(0, \Gamma, \mathbb{D})=2 \omega(0, I, \mathbb{D})=\frac{|I|}{\pi} \leq C(1-d) .
$$

A short computation shows that

$$
\operatorname{diam}(E) \geq C(1-d)\left(1-e^{-\rho_{0}}\right)
$$

for some universal constant C. Since $\mathcal{A}(E)$ is comparable to diam $(E)^{2}$, inequality (2.2) follows.

To use the inequalities (2.1) and (2.2) we shall need some estimates of hyperbolic distance and harmonic measure.

\subsection{Distortion theorems.}

A fundamental result about univalent functions is the Koebe Distortion Theorem. The following estimates, which we have stated in a 
form convenient for our purposes, are easy consequences of this theorem; see $[\mathrm{P}$, pp. 9, 10]. We warn the reader that the hyperbolic metric defined in $[\mathrm{P}]$ differs from $\rho$ by a factor of 2 . For $w \in G$, let $\delta_{G}(w)$ denote the Euclidean distance from $w$ to $\partial G$.

Theorem 2.1 (Koebe). Let $f: \mathbb{D} \longrightarrow G$ be a Riemann map and let $a, b \in G$. Then

$$
\text { 1) } \delta_{G}(f(0)) e^{-\rho_{\mathbb{D}}(0, z)} \leq(1+|z|)^{2}\left|f^{\prime}(z)\right| \leq 4 \delta_{G}(f(0)) e^{3 \rho_{\mathbb{D}}(0, z)},
$$

and

2) $|b-a| \leq 4 \delta_{G}(a) e^{2 \rho_{G}(a, b)}$.

The hyperbolic distance is not explicitly computable in terms of the geometry of $G$ alone. A useful substitute is the quasi-hyperbolic distance on $G$, introduced by Gehring and Palka [GP]. The quasi-hyperbolic distance from $w_{1}$ to $w_{2}$ in $G$ is defined to be

$$
k_{G}\left(w_{1}, w_{2}\right)=\inf \left\{\int_{\gamma} \frac{|d w|}{\delta_{G}(w)}: \quad \gamma \text { is an arc in } G \text { from } w_{1} \text { to } w_{2}\right\} .
$$

It is an easy consequence (see [P, p. 92]) of the Koebe Distortion Theorem that

$$
\frac{1}{2} \rho_{G} \leq k_{G} \leq 2 \rho_{G}
$$

\subsection{Estimates of Harmonic Measure.}

One estimate of harmonic measure which will be used in the proof of Theorem 1.1 is the following Theorem. Let $C_{r}=\{z:|z|=r\}$ be the circle of radius $r$ centered at 0 . If $C_{r} \cap \partial \Omega \neq \varnothing$, define $\theta(r)$ to be the angular measure of the longest component of $C_{r} \cap \Omega$. In other words, $r \theta(r)$ is the length of the longest arc in $C_{r} \cap \Omega$. If $C_{r} \cap \partial \Omega=\varnothing$, set $\theta(r)=\infty$.

Theorem 2.2 (Carleman-Tsuji). For $\varepsilon>0$ and $r>(1+\varepsilon)^{2}|z|$,

$$
\omega\left(z, C_{r}, \Omega\right) \leq C(\varepsilon) \exp \left(-\pi \int_{(1+\varepsilon)|z|}^{r /(1+\varepsilon)} \frac{d r}{r \theta(r)}\right)
$$


where $C(\varepsilon)$ is a constant depending only on $\varepsilon$.

The above result is based on $[\mathrm{C}]$. Tsuji $[\mathrm{T}, \mathrm{p} .116]$ gave the explicit polar coordinate version above, but using the total length of $C_{r} \cap \Omega$, and $2|z|$ for the lower limit in the integral, with $C(\varepsilon)$ comparable to $\varepsilon^{-1 / 2}$. The same proof, using $(1+\varepsilon)|z|$ as the lower limit, gives the result above with $C(\varepsilon)$ comparable to $((1+\varepsilon) / \varepsilon)^{3 / 2}$. Several authors have observed that the proof depends only on the length of the longest arc in $C_{r} \cap \Omega$. See, for instance [HW, p. 123] or [GM], which contains improvements of this theorem.

Another related estimate is based on extremal distance, and is due to Beurling. Let $\Gamma$ be the collection of curves in a region $\Omega$ which connect sets $E \subset \bar{\Omega}$ and $F \subset \bar{\Omega}$. The extremal distance in $\Omega$ from $E$ to $F$ is defined to be

$$
d_{\Omega}(E, F)=\sup _{\lambda} \frac{\left(\inf _{\gamma_{\in} \Gamma} \int_{\gamma} \lambda|d z|\right)^{2}}{\int_{\Omega} \lambda^{2} d \mathcal{A}},
$$

where the supremum is taken over all non-negative Borel functions $\lambda$ with $0<\int_{\Omega} \lambda^{2} d \mathcal{A}<\infty$. Extremal distance is a conformally invariant method of measuring the distance between two sets.

Theorem 2.3 (Beurling). Suppose $\Omega$ is simply connected and $E \subset \bar{\Omega}$. Let $\sigma$ be an arc in $\Omega$ connecting $z_{0}$ to $\partial \Omega$. Then

$$
\omega\left(z_{0}, E, \Omega\right) \leq \frac{8}{\pi} e^{-\pi d_{\Omega}(\sigma, E)}
$$

See, for example [GM]. We will apply this result with $\sigma$ replaced by a disk containing $z_{0}$ which intersects $\partial \Omega$. Since the extremal distance decreases as $\sigma$ is increased, the inequality remains true.

These two preceding theorems are closely related, though there are circumstances where one gives better estimates than the other. For example, the extremal distance between two circles centered at the origin is not changed if radial slits are removed from the region, though this may greatly reduce $\theta(r)$. In this case the Carleman-Tsuji Theorem gives a better estimate. On the other hand, if a curve increasing in modulus and connecting the two bounding circles of the annulus, is removed from the annulus, then the Carleman-Tsuji estimate has $\theta(r)=$ 
$2 \pi$. However, if this curve is not a radial slit, then the Beurling extremal distance estimate gives a better estimate (see the proof below).

The proof of Theorem 1.1 also requires the following elementary estimate. Suppose $\Gamma_{1}$ is a circle orthogonal to $\partial \mathbb{D}$ separating 0 from a set $E \subset \mathbb{D}$, and with $e^{-\rho_{\mathbb{D}}\left(0, \Gamma_{1}\right)} \leq 1 / 4$. If $R=\left[0, e^{i \alpha}\right]$ is the radius orthogonal to $\Gamma_{1}$, let $\Gamma_{0}$ be the circle orthogonal to $\partial \mathbb{D}$ and orthogonal to $R$ with

$$
e^{-\rho_{\mathbb{D}}\left(0, \Gamma_{0}\right)}=2 e^{-\rho_{\mathbb{D}}\left(0, \Gamma_{1}\right)} .
$$

Thus $\Gamma_{0}$ separates 0 from $\Gamma_{1}$ and $E$. Let $\zeta_{0}=\Gamma_{0} \cap R$, so that $\rho_{\mathbb{D}}\left(0, \zeta_{0}\right)=$ $\rho_{\mathbb{D}}\left(0, \Gamma_{0}\right)$.

Proposition 2.4. There is a universal constant $C<\infty$ so that

$$
\sup _{\zeta \in \Gamma_{0}} \omega(\zeta, E, \mathbb{D}) \leq C \omega\left(\zeta_{0}, E, \mathbb{D}\right)
$$

Proof. By conformal invariance, we may suppose that $e^{-\rho_{\mathbb{D}}\left(0, \Gamma_{1}\right)}=$ $1 / 4$ and $\zeta_{0}>0$, which determines $\Gamma_{0}$ and $\Gamma_{1}$. Note that the Euclidean distance from $\Gamma_{0} \cap \mathbb{D}$ to $\Gamma_{1} \cap \mathbb{D}$ is positive. (This is easiest to see using orthogonality and a self-map of the disk which sends $\zeta_{0}=\Gamma_{0} \cap R$ to $0)$. Let $U$ denote the region in $\mathbb{D}$ bounded by $\partial \mathbb{D}$ and $\Gamma_{1}$, containing $\Gamma_{0}$. Let $\varphi$ be a conformal map of $U$ onto $\mathbb{D}$ with $\varphi\left(\zeta_{0}\right)=0$ and set $I=\varphi\left(\Gamma_{1} \cap \mathbb{D}\right) \subset \partial \mathbb{D}$. Note that the Euclidean distance from $\varphi\left(\Gamma_{0} \cap \mathbb{D}\right)$ to $I$ is positive. Since $\omega\left(\varphi^{-1}(z), E, \mathbb{D}\right)$ is a positive harmonic function on $\mathbb{D}$, vanishing on $\partial \mathbb{D} \backslash I$, we have

$$
\omega\left(\varphi^{-1}(z), E, \mathbb{D}\right)=\int_{I} \frac{1-|z|^{2}}{\left|e^{i \theta}-z\right|^{2}} d \mu(\theta),
$$

for some positive measure $d \mu$. Since the distance from $\varphi\left(\Gamma_{0} \cap \mathbb{D}\right)$ to $I$ is positive, if $z \in \varphi\left(\Gamma_{0} \cap \mathbb{D}\right)$ and $e^{i \theta} \in I$, then

$$
\frac{1-|z|^{2}}{\left|e^{i \theta}-z\right|^{2}} \leq C
$$

for some positive constant $C$. Integrating over $I$ proves (2.4), since $\zeta_{0}=\varphi^{-1}(0)$. 


\section{Proof of Theorem 1.1.}

Let $\Omega=f(\mathbb{D})$ and, for $z \in \Omega$, define $\delta_{\Omega}(z)$ to be the Euclidean distance from $z$ to $\partial \Omega$, the boundary of $\Omega$. Multiplying $f$ by a constant, we may assume that $\delta_{\Omega}(0)=1$. For $n \geq 1$, we define

$$
A_{n}=\left\{z:(1+\varepsilon)^{n-1}<|z|<(1+\varepsilon)^{n}\right\}
$$

and $A_{0}=\mathbb{D}$, the unit disk. The size of the annuli was chosen so that the intersection $A_{n} \cap \Sigma_{\varepsilon}$ is roughly a rectangle with Euclidean dimensions comparable to $\varepsilon(1+\varepsilon)^{n-1}$. Choose, if possible, a Euclidean square $Q_{n} \subset A_{n} \cap \Sigma_{\varepsilon} \cap \Omega$ with

$$
\operatorname{diam}\left(Q_{n}\right) \geq \frac{\varepsilon}{4}(1+\varepsilon)^{n-1}
$$

and

$$
\frac{1}{2} \leq \frac{\operatorname{dist}\left(Q_{n}, \partial\left(A_{n} \cap \Sigma_{\varepsilon} \cap \Omega\right)\right)}{\operatorname{diam}\left(Q_{n}\right)} \leq 2 .
$$

Note that there are constants $C_{j}(\varepsilon)$, depending only on $\varepsilon$ so that

$$
C_{1}(\varepsilon) \leq \frac{\operatorname{dist}\left(Q_{n}, \partial \Omega\right)}{\operatorname{diam}\left(Q_{n}\right)} \leq C_{2}(\varepsilon)
$$

Squares satisfying the inequalities in the display above are called Whitney squares. We call the $\left\{Q_{n}\right\}$ dominant Whitney squares, as it turns out that the integral of $|f|$ over their inverse images dominates $\|f\|_{1}$; see Lemma 3.1 below. We remark that many annuli may not contain one of these dominant Whitney squares. Let $z_{n}$ denote the center of $Q_{n}$.

Define a covering $\left\{\Omega_{n}\right\}, n \geq 0$, of $\Omega$ as follows. For $z \in \Omega$, let $\gamma_{z}$ denote the curve from $z$ to 0 , lying on a hyperbolic geodesic. Let $N\left(Q_{n}\right)$ denote the hyperbolic neighborhood of $Q_{n}$ given by

$$
N\left(Q_{n}\right)=\left\{z: \rho_{\Omega}\left(z, z_{n}\right)<\frac{100}{\varepsilon}\right\} .
$$

Put $z$ in $\Omega_{n}$ if $N\left(Q_{n}\right)$ is the first such neighborhood encountered while tracing the path $\gamma_{z}$ starting at $z$. More precisely, $z \in \Omega_{n}$ provided

1) $\rho_{\Omega}\left(z_{n}, \gamma_{z}\right)<100 / \varepsilon$ and 
2) if $\gamma_{z}^{n}$ denotes the component of $\gamma_{z} \backslash N\left(Q_{n}\right)$ containing $z$, then either $\rho_{\Omega}\left(z_{m}, \gamma_{z}^{n}\right) \geq 100 / \varepsilon$ for all $m \neq n$ or else $\gamma_{z}^{n}$ is empty.

If there is no $Q_{n}$ in $A_{n}$, set $\Omega_{n}=\varnothing$.

A few remarks are in order at this point.

i) $N\left(Q_{n}\right) \subset \Omega_{n}$. In particular, the regions $\Omega_{n}$ are not necessarily pairwise disjoint.

ii) $\cup_{n} \Omega_{n}=\Omega$. Since $\delta_{\Omega}(0)=1$, it is easy to check that there is a dominant Whitney square $Q_{0} \subset A_{0}=\mathbb{D}$, with $\operatorname{diam}\left(Q_{0}\right) \geq \varepsilon / 5$. If $z \in Q_{0}$, then $\delta_{\Omega}(z) \geq \varepsilon / 10$, from (3.2). Thus $\delta_{\Omega}(w) \geq \varepsilon / 10$ for $w$ on the radial line segment from 0 to $z$. Integrating the quasi-hyperbolic metric $|d w| / \delta_{\Omega}(w)$ along this segment we have that $\sup _{z \in Q_{0}} \rho_{\Omega}(0, z) \leq 20 / \varepsilon$, and so $N\left(Q_{0}\right)$ contains a neighborhood of 0 . Hence each $\gamma_{z}$ eventually passes through $N\left(Q_{0}\right)$, which means that $\left\{\Omega_{n}\right\}$ covers $\Omega$.

iii) The need for the large hyperbolic radius of $100 / \varepsilon$ will become apparent in the proof, and in Example 4.1 in the last section. It is comparable to the quasi-hyperbolic length in $A_{n}$ of a central circle separating the two bounding circles of $A_{n}$.

Perhaps it is easier to picture the corresponding sets on $\mathbb{D}$. The sets $\left\{f^{-1}\left(N\left(Q_{n}\right)\right)\right\}$ are disks in $\mathbb{D}$. Let $U=\cup_{n} f^{-1}\left(N\left(Q_{n}\right)\right)$. If $z \in \mathbb{D} \backslash U$ then $z \in \Omega_{n}$ if the radial line segment from $z$ to 0 first meets $\partial U$ at a point of $\partial f^{-1}\left(N\left(Q_{n}\right)\right)$.

Since $\cup_{n} Q_{n} \subset \Sigma_{\varepsilon}$ and $\mathbb{D}=\cup_{n} f^{-1}\left(\Omega_{n}\right)$, Theorem 1.1 is as immediate consequence of the following lemma.

Lemma 3.1. There exists a constant $C(\varepsilon)$ such that if $f \in L_{a}^{1}$ is univalent with $f(0)=0$, then

$$
\int_{f^{-1}\left(\Omega_{n}\right)}|f| d \mathcal{A} \leq C(\varepsilon) \int_{f^{-1}\left(Q_{n}\right)}|f| d \mathcal{A} .
$$

Proof. Fix $\varepsilon>0$, with $\varepsilon<1 / 10$. Throughout the proof we will use $C$ to denote various constants that may change from one use to the next, but are independent of any parameters. Similarly, $C(\varepsilon)$ will denote various constants depending only on $\varepsilon$. We emphasize that $C$ and $C(\varepsilon)$ will always be positive.

First we will prove the lemma when $n=0$. We saw in ii) above that $\rho_{\Omega}(0, z) \leq 20 / \varepsilon$, for $z \in Q_{0}$. Thus

$$
\inf _{z \in f^{-1}\left(Q_{0}\right)}(1-|z|) \geq C(\varepsilon)>0 .
$$


By Theorem 2.1.1),

$$
\left|f^{\prime}(z)\right| \leq C(\varepsilon)
$$

on $f^{-1}\left(Q_{0}\right)$. Also by (3.1) and (3.2) we have that $|f|>C(\varepsilon)$ on $Q_{0}$. Combining these observations,

$$
\begin{aligned}
\int_{f^{-1}\left(Q_{0}\right)}|f| d \mathcal{A} & \geq C(\varepsilon) \int_{f^{-1}\left(Q_{0}\right)} d \mathcal{A} \\
& \geq C(\varepsilon) \int_{f^{-1}\left(Q_{0}\right)}\left|f^{\prime}\right|^{2} d \mathcal{A} \\
& =C(\varepsilon) \mathcal{A}\left(Q_{0}\right)
\end{aligned}
$$

and hence

$$
\int_{f^{-1}\left(Q_{0}\right)}|f| d \mathcal{A} \geq C(\varepsilon) .
$$

To estimate the left side of (3.3) when $n=0$, note that by (2.1) and the definition of $A_{j}$,

$$
\int_{f^{-1}\left(\Omega_{0}\right)}|f| d \mathcal{A} \leq C \sum_{j=0}^{\infty}(1+\varepsilon)^{j} e^{-\rho_{\Omega}\left(0, A_{j} \cap \Omega_{0}\right)} \omega\left(0, A_{j} \cap \Omega_{0}, \Omega\right) .
$$

Thus it suffices to show that

$$
e^{-\rho_{\Omega}\left(0, A_{j} \cap \Omega_{0}\right)} \omega\left(0, A_{j} \cap \Omega_{0}, \Omega\right) \leq C(\varepsilon)(1+\varepsilon)^{-j(1+C \varepsilon)} .
$$

Let $f(z) \in A_{j}$. Then, using Theorem 2.1.2) with $a=0$ and the normalization $\delta_{\Omega}(0)=1$,

$$
(1+\varepsilon)^{j-1} \leq|f(z)| \leq 4 e^{2 \rho_{\Omega}(0, f(z))} .
$$

Thus

$$
e^{-\rho_{\Omega}\left(0, A_{j}\right)} \leq C(1+\varepsilon)^{-j / 2} .
$$

To prove the estimate (3.5) we consider several cases. In the first case we will use the extremal length estimate of harmonic measure in Theorem 2.3, in the second case we will estimate harmonic measure using the Carleman-Tsuji Theorem 2.2, and in the remaining case hyperbolic distance alone will increase rapidly enough to obtain (3.5). 
For $1 \leq k \leq j-1$, put $k$ in $\mathcal{K}$ if there exists a component $E_{k}$ of $\partial \Omega \cap A_{k}$ and $\theta_{k} \in[0,2 \pi]$ such that

$$
E_{k} \cap\left\{r e^{i \theta}: 0<r<\infty\right\} \neq \varnothing, \quad \text { when }\left|\theta-\theta_{k}\right| \leq \frac{\varepsilon}{100} .
$$

Case 1. Cardinality $(\mathcal{K}) \geq(j-1) / 3$.

For $k \in \mathcal{K}$, let $S_{k}$ denote a small polar coordinate square centered in the annulus along the ray $\arg z=\theta_{k}$. More precisely, set

$$
S_{k}=\left\{r e^{i \theta}:\left|\theta-\theta_{k}\right|<\frac{\varepsilon}{10^{5}} \text { and }\left|\log \frac{r}{(1+\varepsilon)^{k-1 / 2}}\right|<\frac{\varepsilon}{10^{5}}\right\} .
$$

For $k \notin \mathcal{K}$, set $S_{k}=\varnothing$. We claim that if $\sigma_{k}$ is any curve in $A_{k} \backslash E_{k}$ connecting the two boundary circles of $A_{k}$, then

$$
\int_{\sigma_{k} \backslash S_{k}} \frac{1}{|z|}|d z| \geq \log (1+\varepsilon) .
$$

Inequality (3.8) is clearly true if $\sigma_{k} \cap S_{k}=\varnothing$. If $\sigma_{k} \cap S_{k} \neq \varnothing$, then by (3.7), for at least one component $\sigma_{k}^{\prime}$ of $\sigma_{k} \backslash S_{k}$, we have

$$
\sup _{z, w \in \sigma_{k}^{\prime}}|\arg z-\arg w| \geq \frac{\varepsilon}{100}-\frac{\varepsilon}{10^{5}} .
$$

Thus

$\int_{\sigma_{k} \backslash S_{k}} \frac{1}{|z|}|d z| \geq \sqrt{\left(\log (1+\varepsilon)-\frac{2 \varepsilon}{10^{5}}\right)^{2}+\left(\frac{\varepsilon}{100}-\frac{\varepsilon}{10^{5}}\right)^{2}} \geq \log (1+\varepsilon)$,

which establishes (3.8). The above inequality is perhaps easiest to see by using the change of variable $w=\log z$, so that $|d z| /|z|=|d w|$.

Now define a metric $\lambda$ on $\Omega \cap \cup_{k=1}^{j-1} A_{k}$ by

$$
\lambda(z)=\frac{1}{|z|}\left(\sum_{k=1}^{j-1} \chi_{A_{k}}(z)-\sum_{k \in \mathcal{K}} \chi_{S_{k}}(z)\right)
$$

where $\chi_{F}$ denotes the characteristic function of a set $F$. If $\sigma \subset \Omega$ is a curve connecting $\partial \mathbb{D}$ to $A_{j}$ then by (3.8)

$$
\int_{\sigma} \lambda(z)|d z| \geq(j-1) \log (1+\varepsilon)
$$


Because

$$
\int_{S_{k}} \frac{1}{|z|^{2}} d \mathcal{A}(z) \geq C \varepsilon^{2}
$$

and Cardinality $(\mathcal{K}) \geq(j-1) / 3$, we have that

$$
\int_{\Omega \cap(1+\varepsilon)^{j-1} \mathbb{D}} \lambda^{2}(z) d \mathcal{A}(z) \leq(j-1)\left(2 \pi \log (1+\varepsilon)-C \varepsilon^{2}\right) .
$$

Thus

$$
d_{\Omega}\left(\partial \mathbb{D}, A_{j}\right) \geq \frac{\left(\inf _{\sigma} \int_{\sigma} \lambda(z)|d z|\right)^{2}}{\int_{\Omega} \lambda(z)^{2} d \mathcal{A}} \geq \frac{j-1}{2 \pi}(1+C \varepsilon) \log (1+\varepsilon) .
$$

By Theorem 2.3

$$
\omega\left(0, A_{j} \cap \Omega_{0}, \Omega\right) \leq C e^{-\pi d_{\Omega}\left(\partial \mathbb{D}, A_{j}\right)} \leq C(1+\varepsilon)^{-(j / 2)(1+C \varepsilon)},
$$

and from this and (3.6) we conclude that (3.5) holds in this case.

Recall that $\theta(r)$ is the angular measure of the longest component of $\{z:|z|=r\} \cap \Omega$. For $1 \leq m \leq j-1$, set

$$
F_{m}=\left\{r:(1+\varepsilon)^{m-1}<r<(1+\varepsilon)^{m} \text { and } \theta(r) \leq 2 \pi-\frac{\varepsilon}{100}\right\} .
$$

and

$$
\mathcal{M}=\left\{m: 1 \leq m \leq j-1 \text { and }\left|F_{m}\right| \geq \frac{\varepsilon(1+\varepsilon)^{m-1}}{100}\right\}
$$

Case 2. Cardinality $(\mathcal{M}) \geq(j-1) / 3$.

By the definition of $\overline{\mathcal{M}}$, if $m \in \mathcal{M}$ then

$$
\begin{aligned}
\int_{(1+\varepsilon)^{m-1}}^{(1+\varepsilon)^{m}} \frac{1}{r \theta(r)} d r & \geq \int_{(1+\varepsilon)^{m-1}}^{(1+\varepsilon)^{m}} \frac{1}{2 \pi r} d r+\int_{F_{m}}\left(\frac{1}{2 \pi-\varepsilon / 100}-\frac{1}{2 \pi}\right) \frac{d r}{r} \\
& \geq \frac{1}{2 \pi} \log (1+\varepsilon)+C \varepsilon^{2} .
\end{aligned}
$$

Since Cardinality $(\mathcal{M}) \geq(j-1) / 3$, we have that

$$
\int_{1}^{(1+\varepsilon)^{j-2}} \frac{1}{r \theta(r)} d r \geq \frac{1}{2 \pi}(j-2) \log (1+\varepsilon)+j C \varepsilon^{2} .
$$


Thus by the Carleman-Tsuji Theorem 2.2,

$$
\begin{aligned}
\omega\left(0, A_{j} \cap \Omega_{0}, \Omega\right) & \leq C(\varepsilon) \exp \left(-\pi \int_{1}^{(1+\varepsilon)^{j-2}} \frac{1}{r \theta(r)} d r\right) \\
& \leq C(\varepsilon)(1+\varepsilon)^{-(j / 2)(1+C \varepsilon)} .
\end{aligned}
$$

By (3.6) and (3.10) we conclude that (3.5) holds in this case.

Case 3. Cardinality $(\mathcal{K})<(j-1) / 3$ and Cardinality $(\mathcal{M})<(j-1) / 3$. Set

$$
\mathcal{I}=\{i: 1 \leq i \leq j-1, i \notin \mathcal{K} \text { and } i \notin \mathcal{M}\} .
$$

Then Cardinality $(\mathcal{I}) \geq(j-1) / 3$. Let $i \in \mathcal{I}$. Choose a continuum $L_{i} \subset \partial \Omega \cap A_{i}$ such that $L_{i}$ connects the two bounding circles of $A_{i}$. Since $i \notin \mathcal{K}$, there is a $\theta_{i}$ such that

$$
L_{i} \subset e^{i \theta_{i}} \Sigma_{\varepsilon / 100}
$$

Then $\partial \Omega$ does not intersect most of the middle of $A_{i}$. Indeed, let $V$ denote the annular region given by

$$
V=\left\{z:(1+\varepsilon)^{i-1}\left(1+\frac{\varepsilon}{100}\right)<|z|<(1+\varepsilon)^{i-1}\left(1+\frac{99 \varepsilon}{100}\right)\right\}
$$

and suppose

$$
a \in \partial \Omega \cap\left(V \backslash e^{i \theta_{i}} \Sigma_{\varepsilon / 20}\right) .
$$

Then there is a component $\sigma_{i}$ of $\partial \Omega \cap A_{i}$ connecting $a$ to one of the bounding circles of $A_{i}$. Since $i \notin \mathcal{K}$,

$$
\sigma_{i} \subset e^{i \theta} \Sigma_{\varepsilon / 100}
$$

for some $\theta$. Note that since $i \notin \mathcal{K}$ the angular distance from $\sigma_{i}$ to $L_{i}$ is at least

$$
\frac{\varepsilon}{20}-\left(\frac{\varepsilon}{50}+\frac{\varepsilon}{100}\right)=\frac{\varepsilon}{50} \text {. }
$$

Since the length of $\sigma_{i}$ is at least $(1+\varepsilon)^{i-1} \varepsilon / 100$, this contradicts $i \notin \mathcal{M}$. Thus

$$
\partial \Omega \cap A_{i} \subset e^{i \theta_{i}} \Sigma_{\varepsilon / 20} \cup\left(A_{i} \backslash V\right) .
$$

Since $i \notin \mathcal{M}, \Omega \cap A_{i} \not \subset e^{i \theta_{i}} \Sigma_{\varepsilon / 20}$, and hence there exists a $Q_{i} \in A_{i} \cap \Omega$. 
By (3.6)

$$
\rho_{\Omega}\left(z_{0}, A_{i}\right) \geq \rho_{\Omega}\left(0, A_{i}\right)-\rho_{\Omega}\left(z_{0}, 0\right)>\frac{100}{\varepsilon},
$$

for $i$ sufficiently large. This implies that $A_{i} \cap N\left(Q_{0}\right)=\varnothing$ for $i \geq i_{0}$, where $i_{0}$ depends only on $\varepsilon$.

Suppose $z \in A_{j} \cap \Omega_{0}$ and suppose $\gamma_{z}$ is the curve from $z$ to 0 lying on a hyperbolic geodesic. We claim that if $w \in \gamma_{z} \cap A_{i}$, with $i \geq i_{0}$ and $\delta_{A_{i}}(w) \geq(\varepsilon / 10)(1+\varepsilon)^{i-1}$, then

$$
\delta_{\Omega}(w) \leq \frac{\varepsilon}{10}(1+\varepsilon)^{i-1} .
$$

By (3.1) and (3.2), $\operatorname{dist}\left(Q_{i}, \partial \Omega\right) \geq \varepsilon(1+\varepsilon)^{i-1} / 8$ and hence $Q_{i} \subset$ $V \backslash e^{i \theta_{i}} \Sigma_{\varepsilon / 20}$. Suppose such a $w$ does not satisfy (3.11). Let $\sigma$ be the curve in $V \backslash e^{i \theta_{i}} \Sigma_{\varepsilon / 20}$ connecting $w$ to $z_{i}$ (the center of $Q_{i}$ ), consisting of a radial line segment from $w$ to the circle of radius $(1+\varepsilon)^{i-1}(1+\varepsilon / 2)$, then an arc on this circle, followed by a radial line segment to $z_{i}$. Note that on $\sigma$, the distance to $\partial\left(A_{i} \cap \Omega\right)$ is at least $(\varepsilon / 20)(1+\varepsilon)^{i-1}$, and along most of the circle this distance is $(\varepsilon / 2)(1+\varepsilon)^{i-1}$. Hence by the comparison of the hyperbolic and quasi-hyperbolic distance (2.3)

$$
\rho_{\Omega}\left(w, z_{i}\right) \leq \rho_{A_{i} \cap \Omega}\left(w, z_{i}\right) \leq 2 \int_{\sigma} \frac{|d \zeta|}{\delta_{A_{i} \cap \Omega}(\zeta)}<\frac{100}{\varepsilon} .
$$

Thus $w \in N\left(Q_{i}\right) \cap A_{i}$. Now $A_{i} \cap N\left(Q_{0}\right)=\varnothing$, since $i \geq i_{0}$, and thus $N\left(Q_{0}\right)$ cannot be the first such neighborhood encountered along $\gamma_{z}$. This contradicts $z \in \Omega_{0}$ and completes the proof of (3.11).

Now when $i \geq i_{0}$, by (3.11),

$$
\int_{\gamma_{z} \cap A_{i}} \frac{|d \zeta|}{\delta_{\Omega}(\zeta)} \geq \int_{(1+\varepsilon)^{i-1}(1+\varepsilon / 10)}^{(1+\varepsilon)^{i-1}(1+9 \varepsilon / 10)} \frac{|d \zeta|}{\frac{\varepsilon}{10}(1+\varepsilon)^{i-1}} \geq 8
$$

Using the lower estimate in (2.3), this implies that for $z \in A_{j} \cap \Omega_{0}$,

$$
\rho_{\Omega}(0, z) \geq \frac{1}{2} \sum_{\substack{i \in \mathcal{I} \\ i \geq i_{0}}} \int_{\gamma_{z} \cap A_{i}} \frac{|d \zeta|}{\delta_{\Omega}(\zeta)} \geq 4\left(\frac{j-1}{3}-i_{0}\right) .
$$

Hence

$$
e^{-\rho_{\Omega}\left(0, A_{j} \cap \Omega_{0}\right)} \omega\left(0, A_{j} \cap \Omega_{0}, \Omega\right) \leq e^{-4\left(j-3 i_{0}-1\right) / 3} \leq C(\varepsilon)(1+\varepsilon)^{-j(1+C \varepsilon)},
$$


since $i_{0}$ depends only on $\varepsilon$, and (3.5) holds. This completes the proof of the case $n=0$.

The proof of Lemma 3.1 for $n>0$ is very similar. To begin with, we see from (3.1) that $Q_{n}$ contains a disk of Euclidean radius at least $\varepsilon(1+\varepsilon)^{n-1} / 8 \sqrt{2}$. Thus the quasi-hyperbolic length of any curve $\gamma$ from the center of this disk to its boundary is at least

$$
\int_{\gamma} \frac{|d z|}{\delta_{\Omega}(z)} \geq \frac{C}{(1+\varepsilon)^{n}}|\gamma| \geq C \varepsilon .
$$

Hence $Q_{n}$ contains a hyperbolic ball of radius at least $C \varepsilon$, by $(2.3)$, and so we get from (2.2) that

$$
\begin{aligned}
\int_{f^{-1}\left(Q_{n}\right)}|f| d \mathcal{A} & \geq(1+\varepsilon)^{n-1} \mathcal{A}\left(f^{-1}\left(Q_{n}\right)\right) \\
& \geq C(1+\varepsilon)^{n} \varepsilon^{2} e^{-\rho_{\Omega}\left(0, Q_{n}\right)} \omega\left(0, Q_{n}, \Omega\right) .
\end{aligned}
$$

Next we consider the integral over $f^{-1}\left(\Omega_{n} \cap A_{j}\right)$, where allowance must be made for both $j<n$ and $j \geq n$. We have

$$
\int_{f^{-1}\left(\Omega_{n} \cap A_{j}\right)}|f| d \mathcal{A} \leq(1+\varepsilon)^{j} \mathcal{A}\left(f^{-1}\left(\Omega_{n} \cap A_{j}\right)\right)
$$

$$
\leq C(1+\varepsilon)^{j} e^{-\rho_{\Omega}\left(0, \Omega_{n} \cap A_{j}\right)} \omega\left(0, \Omega_{n} \cap A_{j}, \Omega\right),
$$

by (2.1). Using the triangle inequality and the definition of $\Omega_{n}$, we see that

$$
\rho_{\Omega}\left(0, Q_{n}\right)+\rho_{\Omega}\left(z_{n}, \Omega_{n} \cap A_{j}\right) \leq \rho_{\Omega}\left(0, \Omega_{n} \cap A_{j}\right)+C(\varepsilon),
$$

and hence

$$
e^{-\rho_{\Omega}\left(0, \Omega_{n} \cap A_{j}\right)} \leq C(\varepsilon) e^{-\rho_{\Omega}\left(0, Q_{n}\right)} e^{-\rho_{\Omega}\left(z_{n}, \Omega_{n} \cap A_{j}\right)} .
$$

To estimate $\omega\left(0, \Omega_{n} \cap A_{j}, \Omega\right)$, first suppose that $\rho_{\Omega}\left(0, z_{n}\right) \leq C(\varepsilon)$. We observed above that $Q_{n}$ contains a hyperbolic ball of radius at least $C \varepsilon$, and so $\omega\left(0, Q_{n}, \Omega\right) \geq C(\varepsilon)$. Hence

$$
\omega\left(0, \Omega_{n} \cap A_{j}, \Omega\right) \leq C(\varepsilon) \omega\left(0, Q_{n}, \Omega\right) \omega\left(z_{n}, \Omega_{n} \cap A_{j}, \Omega\right),
$$

by Harnack's inequality. We now show that (3.16) holds for all $n$. We may assume that $\exp \left(-\rho_{\Omega}\left(0, N\left(Q_{n}\right)\right)\right) \leq 1 / 4$, since (3.16) has 
been established when $\rho_{\Omega}\left(0, z_{n}\right) \leq C(\varepsilon)$. Let $\Gamma_{n}$ denote the hyperbolic geodesic in $\mathbb{D}$ that separates 0 from $f^{-1}\left(N\left(Q_{n}\right)\right)$, is orthogonal to the radius of $\mathbb{D}$ through $f^{-1}\left(z_{n}\right)$ and satisfies $\exp \left(-\rho_{\mathbb{D}}\left(0, \Gamma_{n}\right)\right)=$ $2 \exp \left(-\rho_{\mathbb{D}}\left(0, N\left(Q_{n}\right)\right)\right)$. Then

$$
\begin{aligned}
\omega\left(0, \Omega_{n} \cap A_{j}, \Omega\right) & =\omega\left(0, f^{-1}\left(\Omega_{n} \cap A_{j}\right), \mathbb{D}\right) \\
& \leq \omega\left(0, \Gamma_{n}, \mathbb{D}\right) \sup _{\zeta \in \Gamma_{n}} \omega\left(\zeta, f^{-1}\left(\Omega_{n} \cap A_{j}\right), \mathbb{D}\right)
\end{aligned}
$$

by the maximum principle. For the first factor, observe that

$$
\omega\left(0, \Gamma_{n}, \mathbb{D}\right) \leq C(\varepsilon) \omega\left(0, f^{-1}\left(Q_{n}\right), \mathbb{D}\right)=C(\varepsilon) \omega\left(0, Q_{n}, \Omega\right),
$$

since the harmonic measures of these sets in $\mathbb{D}$ are comparable to the diameters of the sets. Next, we use first Proposition 2.4 and then Harnack's inequality to get that

$$
\begin{aligned}
\sup _{\zeta \in \Gamma_{n}} \omega\left(\zeta, f^{-1}\left(\Omega_{n} \cap A_{j}\right), \mathbb{D}\right) & \leq C \omega\left(\zeta_{n}, f^{-1}\left(\Omega_{n} \cap A_{j}\right), \mathbb{D}\right) \\
& \leq C(\varepsilon) \omega\left(f^{-1}\left(z_{n}\right), f^{-1}\left(\Omega_{n} \cap A_{j}\right), \mathbb{D}\right),
\end{aligned}
$$

where $\zeta_{n} \in \Gamma_{n}$ is determined by $\rho_{\mathbb{D}}\left(0, \zeta_{n}\right)=\rho_{\mathbb{D}}\left(0, \Gamma_{n}\right)$. The last three displayed inequalities now combine to complete the proof of (3.16).

Putting together (3.13), (3.14), (3.15) and (3.16), we get that

$$
\begin{aligned}
\int_{f^{-1}\left(\Omega_{n} \cap A_{j}\right)}|f| d \mathcal{A} \leq & C(\varepsilon)(1+\varepsilon)^{j} e^{-\rho_{\Omega}\left(0, Q_{n}\right)} \omega\left(0, Q_{n}, \Omega\right) \\
& \cdot e^{-\rho_{\Omega}\left(z_{n}, \Omega_{n} \cap A_{j}\right)} \omega\left(z_{n}, \Omega_{n} \cap A_{j}, \Omega\right) \\
\leq & C(\varepsilon)(1+\varepsilon)^{j-n} \int_{f^{-1}\left(Q_{n}\right)}|f| d \mathcal{A} \\
& \cdot e^{-\rho_{\Omega}\left(z_{n}, \Omega_{n} \cap A_{j}\right)} \omega\left(z_{n}, \Omega_{n} \cap A_{j}, \Omega\right) .
\end{aligned}
$$

We claim that, for all positive integers $j$ and $n$, we have the inequality

$$
e^{-\rho_{\Omega}\left(z_{n}, \Omega_{n} \cap A_{j}\right)} \omega\left(z_{n}, \Omega_{n} \cap A_{j}, \Omega\right) \leq C(\varepsilon)(1+\varepsilon)^{-|j-n|(1+C \varepsilon)} .
$$

This has been proved when $n=0$ and $z_{n}$ is replaced by 0 , and the proof for $n>0$ is similar. 
For $n>0$ the estimate of hyperbolic distance, as for $n=0$, is based on the Distortion Theorem. Let $z \in A_{j}$, and assume first that $j>n+1$. Then

$$
\begin{aligned}
\frac{\varepsilon(1+\varepsilon)^{j}}{2} & \leq(1+\varepsilon)^{j-1}-(1+\varepsilon)^{n} \\
& \leq\left|z-z_{n}\right| \\
& \leq 4 \delta_{\Omega}\left(z_{n}\right) e^{2 \rho_{\Omega}\left(z_{n}, z\right)} \\
& \leq C \varepsilon(1+\varepsilon)^{n} e^{2 \rho_{\Omega}\left(z_{n}, z\right)},
\end{aligned}
$$

where the upper bound for $\left|z-z_{n}\right|$ came from applying Theorem 2.1.2) with $a=z_{n}$ and $b=z$. For $j<n$ we estimate

$$
\varepsilon(1+\varepsilon)^{n} \leq C \operatorname{diam}\left(Q_{n}\right) \leq C\left|z-z_{n}\right| \leq C(1+\varepsilon)^{j} e^{2 \rho_{\Omega}\left(z_{n}, z\right)},
$$

where now Theorem 2.1.2) was used with $a=z$, noting that $\delta_{\Omega}(z) \leq$ $1+(1+\varepsilon)^{j}$, to get the last inequality. Hence

$$
e^{-\rho_{\Omega}\left(z_{n}, \Omega_{n} \cap A_{j}\right)} \leq C(\varepsilon)(1+\varepsilon)^{-|j-n| / 2}, \quad 0 \leq j<\infty,
$$

after an increase in the constant $C(\varepsilon)$ to handle the cases $j=n$ and $j=n+1$.

The harmonic measure estimates we need for the general case are also very similar to those made in the case $n=0$. As before, we consider cases 1, 2 and 3 separately. The Case 1 estimate involving extremal distance is made in exactly the same way, yielding

$$
\omega\left(z_{n}, \Omega_{n} \cap A_{j}, \Omega\right) \leq C(1+\varepsilon)^{-|j-n|(1+C \varepsilon) / 2},
$$

in place of (3.9). As above, the absolute values are required in the exponent to allow for the possibility that $j<n$.

When we use the Carleman-Tsuji estimate for harmonic measure in Case 2 with $j>n$, the integral in (3.10) is replaced by

$$
\int_{(1+\varepsilon)^{n+1}}^{(1+\varepsilon)^{j-2}} \frac{1}{r \theta(r)} d r .
$$

When $j<n$, we first invert $\Omega$ using the map $z \longrightarrow 1 / z$ (which preserves harmonic measure) to put $\Omega$ in the proper form to apply Theorem 2.2. This results in the estimate

$$
\omega\left(z_{n}, \Omega_{n} \cap A_{j}, \Omega\right) \leq C(\varepsilon)(1+\varepsilon)^{-|j-n|(1+C \varepsilon) / 2},
$$


in place of (3.10).

The estimates (3.19), (3.20), and (3.21) now combine to prove claim (3.18) in cases 1 and 2. Finally, the hyperbolic distance estimate in Case 3 is made just as before to get

$$
\rho_{\Omega}\left(z_{n}, A_{j} \cap \Omega_{n}\right) \geq \frac{4}{3}\left(|j-n|-3 i_{0}-1\right)
$$

instead of (3.12). Hence

$$
e^{-\rho_{\Omega}\left(z_{n}, A_{j} \cap \Omega_{n}\right)} \leq e^{-4\left(|j-n|-3 i_{0}-1\right) / 3} \leq C(\varepsilon)(1+\varepsilon)^{-|j-n|(1+C \varepsilon)},
$$

and (3.18) has been established in this last case as well.

Combining (3.17) and (3.18), we now get

$$
\begin{aligned}
\int_{f^{-1}\left(\Omega_{n}\right)}|f| d \mathcal{A} & =\sum_{j=1}^{\infty} \int_{f^{-1}\left(\Omega_{n} \cap A_{j}\right)}|f| d \mathcal{A} \\
& \leq C(\varepsilon) \int_{f^{-1}\left(Q_{n}\right)}|f| d \mathcal{A} \sum_{j=1}^{\infty}(1+\varepsilon)^{j-n-|j-n|(1+C \varepsilon)} \\
& \leq C(\varepsilon) \int_{f^{-1}\left(Q_{n}\right)}|f| d \mathcal{A}
\end{aligned}
$$

and the proof is complete.

\section{Examples.}

Our first example shows that, in general, infinitely many dominant Whitney squares $Q_{n}$ are required in the proof of Theorem 1.1, and also that $N\left(Q_{n}\right)$ must be defined so that its hyperbolic radius tends to infinity as $\varepsilon \longrightarrow 0$.

EXAmple 4.1. For $R>0$, let $\Omega^{R}=\{z:|z|<R\} \backslash[1, R)$ and let $f_{R}$ be the Riemann map from $\mathbb{D}$ onto $\Omega^{R}$ such that $f_{R}(0)=0$ and $f_{R}^{\prime}(0)>0$. Clearly $f_{R} \in L_{a}^{1}$, and $\lim _{R \rightarrow \infty}\left\|f_{R}\right\|_{1}=\infty$, since as $R \longrightarrow \infty, f_{R}$ converges uniformly on compact subsets of $\mathbb{D}$ to $f(z)=4 z(1+z)^{-2} \notin$ $L_{a}^{1}$. It is clear that there is a dominant Whitney square $Q_{n}$ in every annulus $A_{n}$ with $(1+\varepsilon)^{n} \leq R$. Since

$$
\int_{f_{R}^{-1}\left(Q_{n}\right)}\left|f_{R}\right| d \mathcal{A} \leq(1+\varepsilon)^{n} \mathcal{A}(\mathbb{D})
$$


and $\lim _{R \rightarrow \infty}\left\|f_{R}\right\|_{1}=\infty$, as $R \longrightarrow \infty$ we must use $Q_{j}$ with $j$ arbitrarily large in the proof of Theorem 1.1.

Next, we show that it was necessary to have the hyperbolic radius of $N\left(Q_{n}\right)$ tending to infinity as $\varepsilon \longrightarrow 0$. We show that if $M>0$ is any fixed constant, then the neighborhoods $\mathcal{N}\left(Q_{n}\right)=\left\{z: \rho_{\Omega}\left(z, z_{n}\right)<M\right\}$ will not work in the proof. Let $z \in \Omega^{R}$ be a point with $\operatorname{Re} z<0$. Then $\left|z_{n}-z\right| \geq\left|z_{n}\right|$, and $\delta\left(z_{n}\right)$ is comparable to $\varepsilon\left|z_{n}\right|$, and so

$$
\frac{1}{\varepsilon} \leq C \frac{\left|z_{n}-z\right|}{\delta_{\Omega}\left(z_{n}\right)} \leq C e^{2 \rho_{\Omega}\left(z_{n}, z\right)}
$$

by Theorem 2.1.2). Thus, if $\varepsilon$ is sufficiently small, independent of $R$, then $\mathcal{N}\left(Q_{n}\right)$ is contained in the right half plane for all $n \geq 0$. This means that if $\left(\Omega^{R}\right)_{n}$ is defined using $\mathcal{N}\left(Q_{n}\right)$ in place of $N\left(Q_{n}\right)$, then $\left\{\left(\Omega^{R}\right)_{n}\right\}$ does not cover $\Omega^{R}$, and so the proof of Theorem 1.1 does not work. We now show that even if $Q_{0}$ is replaced by $\mathbb{D} \cap \Sigma_{\varepsilon}$, so that now $\left\{\left(\Omega^{R}\right)_{n}\right\}$ covers $\Omega^{R}$, there still is a problem.

Since $f_{R}$ maps $(-1,0]$ to $(-R, 0]$, it follows that a hyperbolic neighborhood in $\mathbb{D}$ of $(-1,-1 / 2]$ must belong to $f_{R}^{-1}\left(\left(\Omega^{R}\right)_{0}\right)$, when $\left(\Omega^{R}\right)_{n}$ is defined using $\mathcal{N}\left(Q_{n}\right)$ in place of $N\left(Q_{n}\right)$. This hyperbolic neighborhood contains an angle

$$
\Gamma=\left\{z \in \mathbb{D}:|1+z|<(1+\eta)(1-|z|) \text { and }|1+z|<\frac{1}{2}\right\}
$$

where $\eta>0$, in $\mathbb{D}$ with vertex at -1 . Since $f_{R}$ converges uniformly on compact subsets of $\mathbb{D}$ to $4 z(1+z)^{-2}$, which has a pole of order 2 at -1 , it follows that

$$
\lim _{R \rightarrow \infty} \int_{f_{R}^{-1}\left(\left(\Omega^{R}\right)_{0}\right)}\left|f_{R}\right| d \mathcal{A} \geq \lim _{R \rightarrow \infty} \int_{\Gamma}\left|f_{R}\right| d \mathcal{A}=\infty .
$$

Thus, if $N\left(Q_{n}\right)$ is replaced by $\mathcal{N}\left(Q_{n}\right)$, then there is no constant $C(\varepsilon)$ depending only on $\varepsilon$ such that

$$
\int_{f_{R}^{-1}\left(\left(\Omega^{R}\right)_{0}\right)}\left|f_{R}\right| d \mathcal{A} \leq C(\varepsilon) \int_{f_{R}^{-1}\left(Q_{0}\right)}\left|f_{R}\right| d \mathcal{A},
$$

since the integral on the right is bounded by $1 \cdot \mathcal{A}(\mathbb{D})=\pi$.

EXAMPLE 4.2. It might seem at first thought that the proof of Theorem 1.1 could be simplified by using circular symmetrization. However, this 
does not seem to be the case. One problem is that the symmetrization of an $L_{a}^{1}$ function may not be in $L_{a}^{1}$. For example, let $g(z)=\left(f\left(z^{2}\right)\right)^{1 / 2}$ be the square root transform of the function $f$ from Example 4.1. Then $g(z)=2 z /\left(1+z^{2}\right)$, and $g$ maps the disk onto the plane slit along the real axis from $-\infty$ to -1 and from 1 to $\infty$. Clearly $g \in L_{a}^{1}$, since its poles are simple, but its circular symmetrization is $f \notin L_{a}^{1}$.

Let $g_{R}$ be the Riemann map of $\mathbb{D}$ onto

$$
\{z:|z|<R\} \backslash((-R,-1] \cup[1, R))
$$

with

$$
g_{R}(0)=0
$$

and

$$
g_{R}^{\prime}(0)>0 .
$$

Then $\left\|g_{R}\right\|_{1} \leq\|g\|_{1}$, since $g_{R}$ is subordinant to $g$, and so the integrals of $\left|g_{R}\right|$ over the inverse image of $\Sigma_{\varepsilon}$ are uniformly bounded by $\|g\|_{1}$. The symmetrization of $g_{R}$ is $f_{R}$, and

$$
\lim _{R \rightarrow \infty} \int_{f_{R}^{-1}\left(\Sigma_{\varepsilon}\right)}\left|f_{R}\right| d \mathcal{A} \geq \lim _{R \rightarrow \infty} \delta \int_{\mathbb{D}}\left|f_{R}\right| d \mathcal{A}=\infty
$$

where Theorem 1.1 was used to get the inequality. Thus even when the symmetrized function is in $L_{a}^{1}$, its integral over the inverse image of $\Sigma_{\varepsilon}$ cannot be bounded by the integral of the original function.

ExAmple 4.3. This example shows that in Theorem 1.1, $L_{a}^{1}$ can not be replaced by $L_{a}^{p}$, for any $p>1$. Let $p>1$ be fixed and set

$$
\varepsilon_{n}=\pi\left(\frac{p-1}{p}\right)+\frac{1}{n}
$$

for all $n$ sufficiently large so that $\varepsilon_{n} \leq \pi$. Let

$$
f_{n}: \mathbb{D} \longrightarrow \mathbb{C} \backslash\left(1+\Sigma_{\varepsilon_{n}}\right)
$$

be the Riemann map with $f_{n}(0)=0$ and $f_{n}^{\prime}(0)>0$. It is easy to verify that $f_{n} \in L_{a}^{p}$, since $\varepsilon_{n}>\pi(p-1) / p$, but

$$
\left\|f_{n}\right\|_{p} \longrightarrow \infty, \quad \text { as } n \longrightarrow \infty \text {. }
$$


On the other hand, some elementary trigonometry shows that

$$
\Sigma_{\pi(p-1) / 2 p} \cap\left(\mathbb{C} \backslash\left(1+\Sigma_{\pi(p-1) / p}\right)\right)
$$

is contained in the disk of radius 2 centered at the origin. Hence

$$
\int_{f_{n}^{-1}\left(\Sigma_{\pi(p-1) / 2 p}\right)}\left|f_{n}\right|^{p} d \mathcal{A} \leq 2^{p} \mathcal{A}(\mathbb{D})=2^{p} \pi
$$

and this can not be used to dominate $\left\|f_{n}\right\|_{p}$ as $n \longrightarrow \infty$.

\section{References.}

[A1] Ahlfors, L. V., Lectures on quasiconformal mappings. Van Nostrand, 1966.

[A2] Ahlfors, L. V., Conformal Invariants: Topics in Geometric Function Theory. McGraw-Hill, 1973.

[C] Carleman, T., Sur une inégalité différentielle dans la théorie des fonctions analytiques. C. R. Acad. Sci. Paris 196 (1933), 995-997.

[GM] Garnett, J. B., Marshall, D. E., Harmonic Measure. Cambridge Univ. Press. Forthcoming.

[GP] Gehring, F. W., Palka, B. P., Quasiconformally homogeneous domains. J. Analyse Math. 30 (1976), 172-199.

[Ha] Hamilton, R. S., Extremal quasiconformal mappings with prescribed boundary values. Trans. Amer. Math. Soc. 138 (1969), 399-406.

[HW] Hayman, W. K., Weitsman, A., On the coefficients and means of functions omitting values. Math. Proc. Cambridge Philos. Soc. 77 (1975), 119-137.

[Hu] Huang, X., The image domain of an extremal dilatation. Adv. in Math. (China) 22 (1993), 435-440.

[K] Krushkal, S. L., Teichmüller's theorem on extremal quasi-conformal mappings. Siberian Math. J. 8 (1967), 231-244. Translated from Sibirsk. Math. Z. 8 (1967), 313-332.

[OS] Ortel, M., Smith, W., The argument of an extremal dilatation. Proc. Amer. Math. Soc. 104 (1988), 498-502.

[P] Pommerenke, Ch., Boundary Behavior of Conformal Maps. SpringerVerlag, 1992.

[RS] Reich, E., Strebel, K., Extremal quasiconformal mappings with given boundary values. Contributions to Analysis, a collection of papers dedicated to Lipman Bers. Academic Press, 1974, 375-391. 
[S] Strebel, K., On quadratic differentials and extremal quasiconformal mappings. Proc. Internat. Congress Math. Vancouver, 1974. Canad. Math. Congr. 2 (1975), 223-227.

[T] Tsuji, M., Potential Theory in Modern Function Theory. Chelsea, 1959.

Recibido: 19 de septiembre de 1.997

\author{
Donald E. Marshall* \\ Department of Mathematics \\ Box 354350 \\ University of Washington \\ Seattle, Washington, 98195-4350, USA \\ marshall@math. washington. edu \\ and \\ Wayne Smith ${ }^{\dagger}$ \\ Department of Mathematics \\ University of Hawaii \\ Honolulu, Hawaii 96822, USA \\ wayne@math .hawaii. edu
}

* Supported in part by NSF grant DMS-9532078.

$\dagger$ Supported in part by the Milliman fund. 